\title{
CLINICOPATHOLOGICAL STUDY OF LIVER ABSCESS IN EASTERN INDIAN POPULATION
}

Himansu Roy1, Ashish Ranjan²

${ }^{1}$ Associate Professor, Department of General Surgery, Medical College and Hospital, Kolkata, West Bengal, India.

${ }^{2}$ Senior Resident, Department of General Surgery, Islampur SS/SD Hospital, Uttar Dinajpur, West Bengal, India.

ABSTRACT

\section{BACKGROUND}

A descriptive study of Liver abscess is defined as collection of purulent material in liver parenchyma. It could be amoebic, pyogenic, infected hydatid, traumatic or ascaridal. It is a burning problem in tropical nations with often lethal consequences and diagnostic/therapeutic challenges.

This study aims to find out the demographic distribution, different modes of presentations, risk factors association, alteration of various laboratory and radiological investigations in diagnosis of liver abscess and responses to standard institutional management strategies.

\section{MATERIALS AND METHODS}

Medical records were taken by preformed case, Proforma. Descriptive statistics were used and analysed by SPSS software.

\section{RESULTS}

3/4th of cases belonged to 4th to 6th decade. Males were more commonly affected than females. Alcoholism was the most consistent risk factor followed by diabetes mellitus. Most common presenting symptom and sign were abdominal pain and abdominal tenderness in right hypochondriac region respectively. Most common aetiology of liver abscess was amoebic followed by mixed amoebic and pyogenic infections. The most common organisms obtained from pus C/S from liver abscess were Enterococcus spp. and Escherichia coli. Abnormal chest x-ray findings were recorded in $>50 \%$ of cases. USG showed majority of cases had large, solitary liver abscess in right lobe of liver. Most common complication observed was intraabdominal rupture of liver abscess, for which open surgical drainage was performed.

\section{CONCLUSION}

Recurrence was observed more following conservative management than other modalities.

\section{KEY WORDS}

Liver Abscess, Pyogenic Abscess, Amoebic Abscess, Percutaneous Drainage, Recurrence.

HOW TO CITE THIS ARTICLE: Roy H, Ranjan A. Clinicopathological study of liver abscess in eastern Indian population. J. Evolution Med. Dent. Sci. 2018;7(39):4307-4312, DOI: 10.14260/jemds/2018/960

\section{BACKGROUND}

- A descriptive study of Liver is the organ most subjected to the development of abscess. Liver abscess accounts for $48 \%$ of all visceral abscesses.

- India has $2^{\text {nd }}$ highest incidence of liver abscess in the world.(1)

- $\quad$ Liver abscess associated with mortality upto $20 \%$.(2) $^{(2)}$

- Three major forms of liver abscess are:

- Pyogenic- $3 / 4^{\text {th }}$ of liver abscess in developed countries.

- Amoebic- $2 / 3^{\text {rd }}$ of liver abscess in developing countries.

- Fungal- Less common.

- In the recent past, the incidence of liver abscess has increased.

- There has been a changing trend in demographic presentation, clinical profile, microbiological aetiology, management strategies and outcomes of patients diagnosed with liver abscess.

'Financial or Other Competing Interest': None.

Submission 21-06-2018, Peer Review 25-07-2018,

Acceptance 01-08-2018, Published 24-09-2018.

Corresponding Author:

Dr. Ashish Ranjan,

Department of Horticulture (Veg. and Flori.)

Bihar Agricultural University,

Sabour, Bhagalpur-813210, India.

E-mail: drashishranjan2209@gmail.com

DOI: $10.14260 /$ jemds $/ 2018 / 960$
- The concept of the present study is to find out the demographic distribution, different modes of presentations, risk factors association, alteration of various laboratory and radiological investigations in diagnosis of liver abscess and responses to standard institutional management strategies.

\section{Objectives/ Primary Objective}

To Study Liver Abscess patients with respect to-

1. Demographic profile of patients.

2. Spectrum of clinical presentations.

3. Risk factors association.

4. Alteration in various blood and radiological investigations.

5. Bacteriological and serological characteristics.

\section{Secondary Objective}

To evaluate the response to standard institutional management strategies.

\section{MATERIALS AND METHODS}

- Place of study: Medical college and Hospital, Kolkata.

- Duration of study: January 2016 to June 2017.

- Design of study: A descriptive study.

- Study Population: Indoor patients of Liver Abscess admitted in Department of General Surgery, Medical College and Hospital, Kolkata. 


\section{Statistical Analysis}

The qualitative data was expressed by Fisher's test, Independent t-test. For statistical analysis, SPSS software version 25.0 was used.

\section{Inclusion Criteria}

- All cases of liver abscess diagnosed clinically and/or ultrasonographically.

- All cases of bacterial and parasitic liver abscess.

\section{Exclusion Criteria}

- Traumatic liver abscess.

- Liver abscess associated with liver malignancy.

\section{Sample Size}

The descriptive study was carried out in the Medical College and Hospital, Kolkata where a total of 50 patients' based on previous year records were taken. Sample size was taken for convenience.

\section{Data Analysis}

- $\quad$ By following standard statistical protocols, all data will be collected and analysed to evaluate the clinicopathological aspects and response to standard institutional management strategies.

- Continuous data was summarised as Mean \pm SD (standard deviation of the mean), while categorical data was represented in numbers and percentages. Analyses were performed on SPSS software (Windows version 17.0).

\section{RESULTS}

Demographic Features

There were $45(90 \%)$ males and $5(10 \%)$ females.

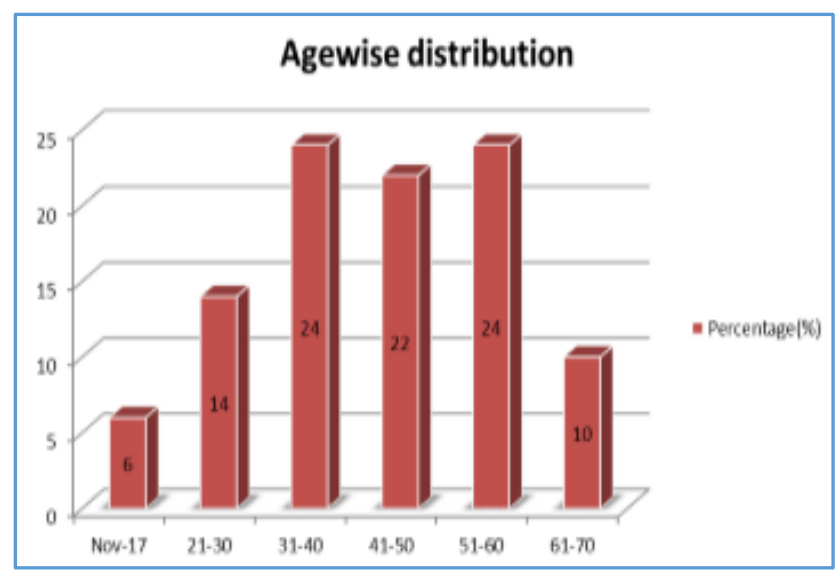

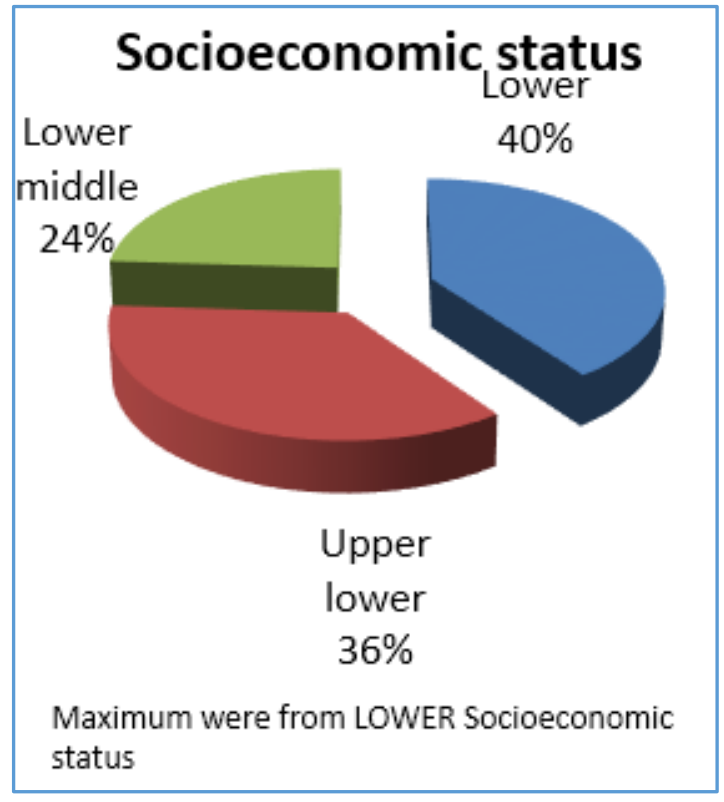

\section{Clinical History}

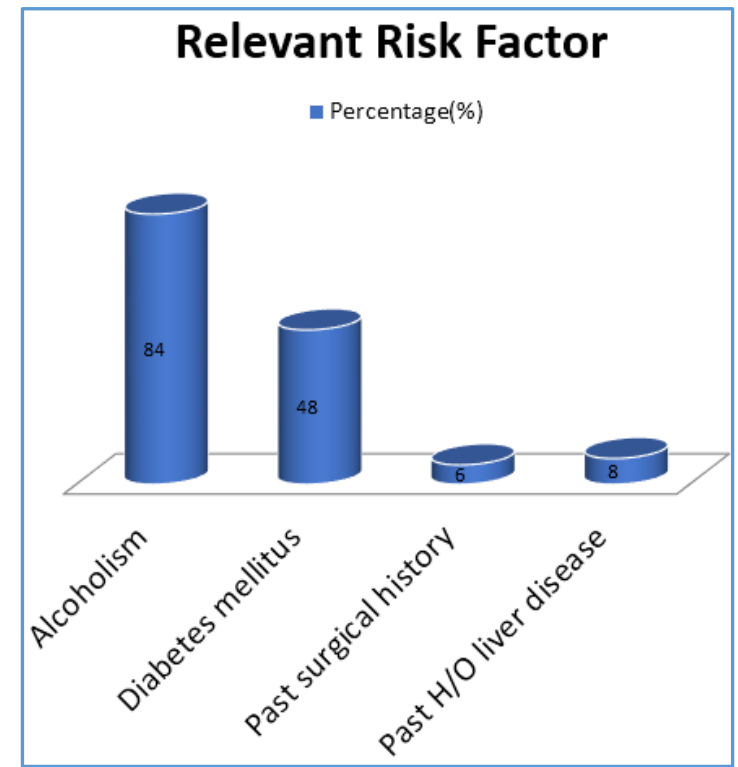

Most Common: Alcoholism (84\%)

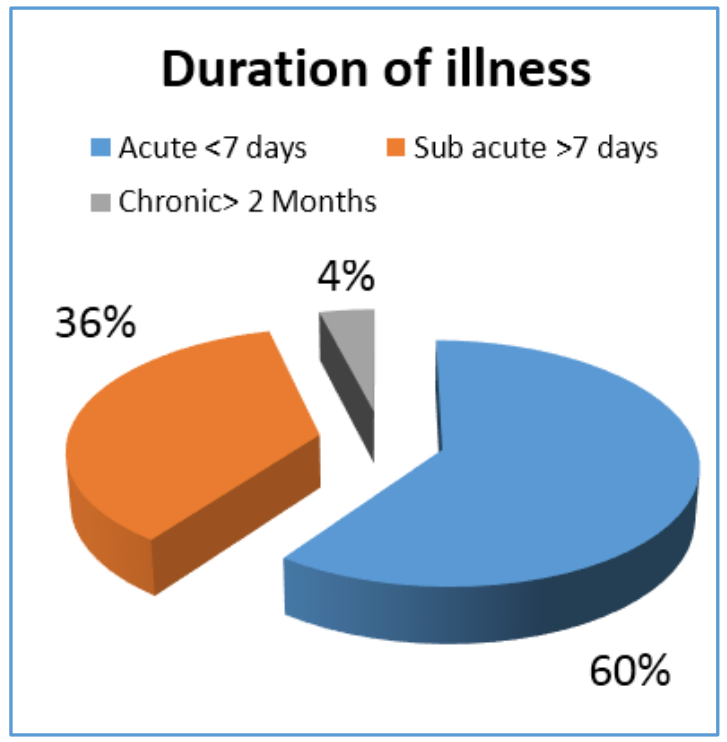

Majority presented Acutely within 7 Days 


\begin{tabular}{|c|c|c|}
\hline Symptoms and Signs & Number (N) & Percentage (\%) \\
\hline Pain in abdomen & 50 & 100 \\
\hline Fever $\left(>38.5^{\circ} \mathrm{C}\right)$ & 47 & 94 \\
\hline Abdominal tenderness & 50 & 100 \\
\hline Hepatomegaly & 25 & 50 \\
\hline Icterus & 24 & 48 \\
\hline Cough & 15 & 30 \\
\hline Pallor & 12 & 24 \\
\hline Loose stools & 8 & 16 \\
\hline Altered sensorium & 2 & 4 \\
\hline \multicolumn{2}{|c|}{ Clinical Manifestations } \\
\hline
\end{tabular}

Radiological Analysis

\begin{tabular}{|c|c|c|}
\hline Chest X-ray Findings & N & $\mathbf{\%}$ \\
\hline \multicolumn{1}{|c|}{ Normal } & 19 & 38 \\
\hline$\bullet \quad \begin{array}{l}\text { Elevated right dome of } \\
\text { diaphragm }\end{array}$ & 31 & 62 \\
\hline$\bullet \quad$ Pleural effusion & 23 & 46 \\
\hline$\bullet \quad$ Right basal pneumonitis & 12 & 24 \\
\hline$\bullet \quad$ Air fluid level & 10 & 20 \\
\hline
\end{tabular}

\begin{tabular}{|c|c|c|c|}
\hline Inv. & Abnormality & $\mathbf{N}$ & $\%$ \\
\hline \multirow{3}{*}{ Routine } & Leucocytosis (> 11,000 cells/cumm) & 34 & 68 \\
\hline & Anaemia $(<10 \mathrm{~g} / \mathrm{dL})$ & 11 & 22 \\
\hline & Hyperglycaemia (> $200 \mathrm{mg} / \mathrm{dL}$ ) & 28 & 56 \\
\hline \multirow{2}{*}{ RFT } & Raised urea $(>45 \mathrm{mg} / \mathrm{dL})$ & 12 & 24 \\
\hline & Raised creatinine (>1.2 mg/dL) & 9 & 18 \\
\hline \multirow{6}{*}{ LFT } & Hyperbilirubinaemia (> $2.4 \mathrm{mg} / \mathrm{dL}$ ) & 29 & 58 \\
\hline & Hypoalbuminaemia $(<3.5 \mathrm{mg} / \mathrm{dL})$ & 42 & 84 \\
\hline & Elevated Sr. ALP (> $150 \mathrm{IU} / \mathrm{L})$ & 42 & 84 \\
\hline & Elevated SGOT (> $40 \mathrm{IU} / \mathrm{L})$ & 24 & 48 \\
\hline & Elevated SGPT (> $40 \mathrm{IU} / \mathrm{L})$ & 37 & 74 \\
\hline & $\begin{array}{c}\text { Elevated prothrombin time (> } 14 \\
\text { secs) }\end{array}$ & 24 & 48 \\
\hline \multicolumn{4}{|c|}{ Biochemical Analysis } \\
\hline
\end{tabular}

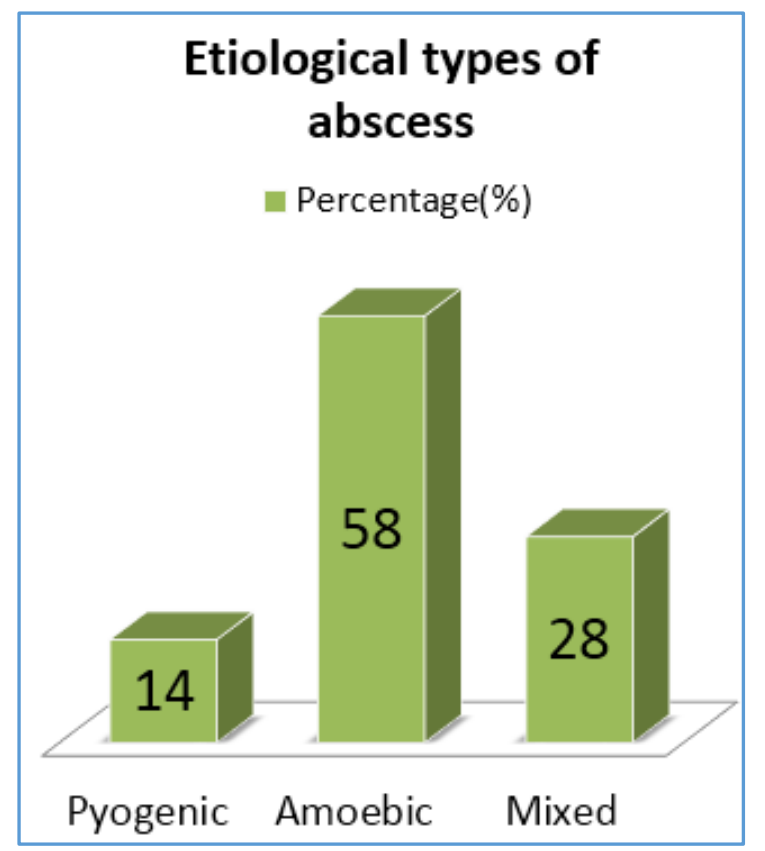

\begin{tabular}{|c|c|c|}
\hline USG Findings & N & \% \\
\hline Lobe involved & 37 & 74 \\
Right & 6 & 12 \\
Left & 7 & 14 \\
Both & 37 & 74 \\
\hline Number of abscesses & 13 & 26 \\
Solitary & 16 & 32 \\
Multiple & 34 & 68 \\
\hline Size of abscesses & & \\
Small (<5 cms) & & \\
Large (>5 cms) &
\end{tabular}

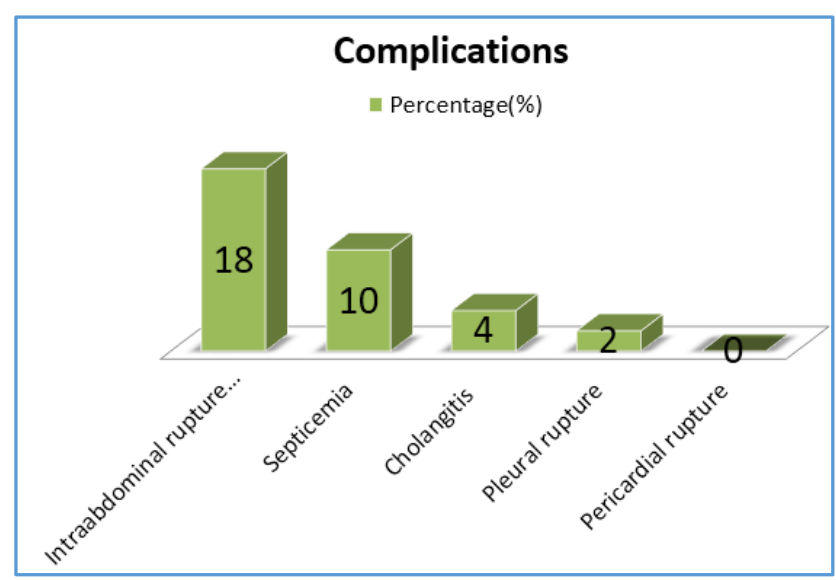

Complications of Liver Abscess

\begin{tabular}{|c|c|c|c|}
\hline Type of Abscess & Treatment Modality & $\mathbf{N}$ & $\%$ \\
\hline Small $(<5 \mathrm{cms}$ ) & Medical (antibiotics) & 12 & 24 \\
\hline Large (> $5 \mathrm{cms}$ ) & $\begin{array}{c}\text { USG-guided pigtail } \\
\text { drainage + antibiotics }\end{array}$ & 29 & 58 \\
\hline $\begin{array}{l}\text { Intraabdominal } \\
\text { ruptured abscess }\end{array}$ & Open surgical drainage & 9 & 18 \\
\hline & Total & 50 & 100 \\
\hline
\end{tabular}

Microbiological Analysis

\begin{tabular}{|c|c|c|}
\hline Pus Culture & N & \% \\
\hline Enterococcus spp. & 7 & 18 \\
\hline E. coli & 7 & 18 \\
\hline K. pneumoniae & 4 & 11 \\
\hline S. aureus & 3 & 8 \\
\hline No growth & 17 & 45 \\
\hline Total & $\mathbf{3 8}$ & $\mathbf{1 0 0}$ \\
\hline
\end{tabular}

\begin{tabular}{|c|c|c|}
\hline Amoebic Serology (IgG) & $\mathbf{N}$ & $\mathbf{\%}$ \\
\hline Positive & 43 & 86 \\
\hline Negative & 7 & 14 \\
\hline Total & $\mathbf{5 0}$ & $\mathbf{1 0 0}$ \\
\hline
\end{tabular}

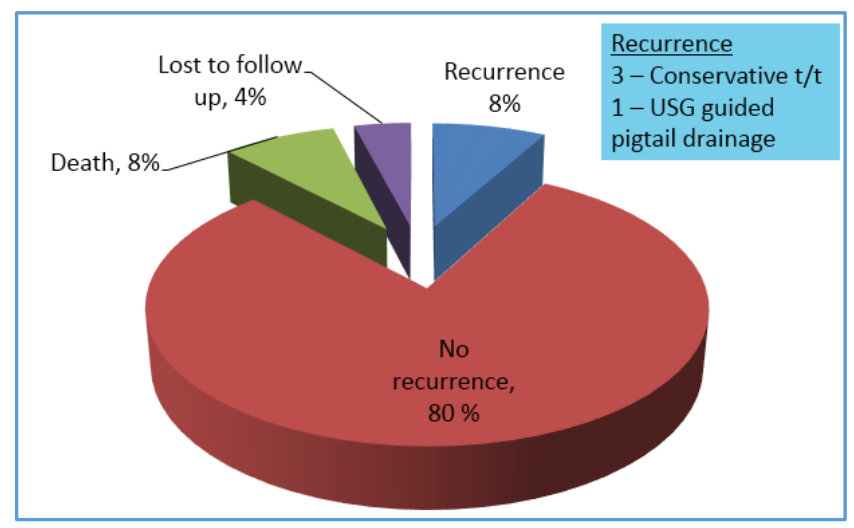

Follow-Up and Recurrence 
I. Demographic Features

\begin{tabular}{|c|c|c|}
\hline Studies & Male & Female \\
\hline Present Study & $90 \%$ & $10 \%$ \\
\hline Santos Rosa et al(3) & $70 \%$ & $30 \%$ \\
\hline Venu Gopal et al(4) & $65.1 \%$ & $34.9 \%$ \\
\hline \multicolumn{3}{|c|}{ Gender } \\
\hline
\end{tabular}

\begin{tabular}{|c|c|c|c|c|c|}
\hline \multicolumn{2}{|c|}{ Studies } & \multicolumn{2}{|c|}{$\begin{array}{c}\text { Most Common Age } \\
\text { Group Affected (yrs.) }\end{array}$} & \multicolumn{2}{|c|}{$\begin{array}{c}\text { Range of Ages } \\
\text { (yrs.) }\end{array}$} \\
\hline \multicolumn{2}{|c|}{$\begin{array}{l}\text { Venu Gopal } \\
\text { et al }\end{array}$} & \multicolumn{2}{|r|}{$20-50$} & \multicolumn{2}{|c|}{$20-75$} \\
\hline \multicolumn{2}{|c|}{ Chaudhary et al(5) } & \multicolumn{2}{|r|}{$31-60$} & \multicolumn{2}{|c|}{$19-69$} \\
\hline \multicolumn{2}{|c|}{ Jha et al(6) } & \multicolumn{2}{|r|}{$21-50$} & \multicolumn{2}{|c|}{$2.5-70$} \\
\hline \multicolumn{2}{|c|}{ Present Study } & \multicolumn{2}{|r|}{$31-60$} & \multicolumn{2}{|c|}{$15-69$} \\
\hline \multicolumn{6}{|c|}{ Age } \\
\hline Studies & Lower & $\begin{array}{l}\text { Upper } \\
\text { Lower }\end{array}$ & \begin{tabular}{l|l} 
Lower & \\
Middle & 1 \\
\end{tabular} & $\begin{array}{l}\text { Upper } \\
\text { Middle }\end{array}$ & Upper \\
\hline $\begin{array}{l}\text { Present } \\
\text { Study }\end{array}$ & $20(40 \%)$ & $18(36 \%$ & 6) $12(24 \%) \mid$ & - & - \\
\hline \multicolumn{6}{|c|}{ Socioeconomic Status } \\
\hline Risk Factor & \multicolumn{2}{|c|}{\begin{tabular}{|l|l} 
Present Study & I \\
\end{tabular}} & Mathur et al(7) & \multicolumn{2}{|c|}{ Chan et al(8) } \\
\hline Alcoholism & \multicolumn{2}{|c|}{$84 \%$} & $70 \%$ & \multicolumn{2}{|r|}{ 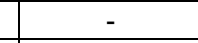 } \\
\hline $\begin{array}{l}\text { Diabetes } \\
\text { Mellitus }\end{array}$ & \multicolumn{2}{|c|}{$48 \%$} & - & \multicolumn{2}{|c|}{$59.8 \%$} \\
\hline
\end{tabular}

\begin{tabular}{|c|c|c|c|}
\hline Studies & Acute & Subacute & Chronic \\
\hline Present study & $30(60 \%)$ & $18(36 \%)$ & $2(4 \%)$ \\
\hline Kapadia et al $(9)$ & & Mostly & \\
\hline \multicolumn{2}{|c|}{ Onset of Disease and Hospital Presentation } \\
\hline
\end{tabular}

\section{Clinical Features}

\begin{tabular}{|c|c|c|c|c|}
\hline Symptom & $\begin{array}{c}\text { Mangukiya } \\
\text { et al(10) }\end{array}$ & $\begin{array}{c}\text { Santos Rosa } \\
\text { et al(3) }\end{array}$ & $\begin{array}{c}\text { Chaudhary } \\
\text { et al(5) }\end{array}$ & $\begin{array}{c}\text { Present } \\
\text { Study }\end{array}$ \\
\hline $\begin{array}{c}\text { Pain in } \\
\text { abdomen }\end{array}$ & $97 \%$ & $90 \%$ & $98 \%$ & $100 \%$ \\
\hline Fever & $74 \%$ & $40 \%$ & $94 \%$ & $90 \%$ \\
\hline Cough & $14 \%$ & - & $35 \%$ & $30 \%$ \\
\hline Icterus & $26.5 \%$ & $70 \%$ & $25 \%$ & $48 \%$ \\
\hline Loose stools & $6.2 \%$ & $10 \%$ & $18 \%$ & $16 \%$ \\
\hline $\begin{array}{c}\text { Altered } \\
\text { sensorium }\end{array}$ & $4.5 \%$ & $0 \%$ & $0 \%$ & $4 \%$ \\
\hline \multicolumn{5}{|c|}{ Symptoms } \\
\hline
\end{tabular}

\begin{tabular}{|c|c|c|c|}
\hline Signs & $\begin{array}{c}\text { Mangukiya } \\
\text { et al(11) }\end{array}$ & $\begin{array}{c}\text { Chaudhary } \\
\text { et al(6) }\end{array}$ & $\begin{array}{c}\text { Present } \\
\text { Study }\end{array}$ \\
\hline Jaundice & - & $24 \%$ & $24 \%$ \\
\hline Pallor & - & $23 \%$ & $24 \%$ \\
\hline Fever $\left(>38.5^{\circ} \mathrm{C}\right)$ & $74 \%$ & $94 \%$ & $94 \%$ \\
\hline Abdominal tenderness & $95 \%$ & $100 \%$ & $100 \%$ \\
\hline Hepatomegaly & $26 \%$ & $50 \%$ & $50 \%$ \\
\hline Respiratory findings & $14 \%$ & $61 \%$ & $62 \%$ \\
\hline Shock & $4.5 \%$ & $0 \%$ & $4 \%$ \\
\hline \multicolumn{3}{|c}{ Signs } \\
\hline
\end{tabular}

\begin{tabular}{|c|c|c|c|c|}
\hline Investigation & $\begin{array}{c}\text { Mangukiya } \\
\text { et al(9) }\end{array}$ & $\begin{array}{c}\text { Jha } \\
\text { et al(5) }\end{array}$ & $\begin{array}{c}\text { Santos } \\
\text { Rosa } \\
\text { et al(2) }\end{array}$ & $\begin{array}{c}\text { Present } \\
\text { Study }\end{array}$ \\
\hline $\begin{array}{c}\text { Leucocytosis } \\
(>11,000 / \text { cumm })\end{array}$ & $78 \%$ & $75 \%$ & $89 \%$ & $68 \%$ \\
\hline $\mathrm{Hb}(<11 \mathrm{~g} / \mathrm{dL})$ & $41.5 \%$ & $52 \%$ & $0 \%$ & $22 \%$ \\
\hline Hyperbilirubinaemi & $12.5 \%$ & $18 \%$ & $75 \%$ & $58 \%$ \\
\hline
\end{tabular}

\begin{tabular}{|c|c|c|c|c|}
\hline a & & & & \\
\hline Elevated PT & $34.5 \%$ & $43 \%$ & $75 \%$ & $48 \%$ \\
\hline Hypoalbuminaemia & - & $40 \%$ & $80 \%$ & $84 \%$ \\
\hline Raised ALP & - & $63 \%$ & $100 \%$ & $84 \%$ \\
\hline Elevated SGOT & - & $48 \%$ & $88 \%$ & $48 \%$ \\
\hline Elevated SGPT & - & $50 \%$ & $88 \%$ & $74 \%$ \\
\hline \multicolumn{5}{|c|}{ Biochemical Analysis } \\
\hline
\end{tabular}

\begin{tabular}{|c|c|c|c|c|}
\hline Organisms & $\begin{array}{c}\text { Czerwonko } \\
\text { et al(11) }\end{array}$ & $\begin{array}{c}\text { Peris } \\
\text { et al(12) }\end{array}$ & $\begin{array}{c}\text { Kong } \\
\text { et al(13) }\end{array}$ & $\begin{array}{c}\text { Present } \\
\text { Study }\end{array}$ \\
\hline E. coli & $26.7 \%$ & $16.3 \%$ & $9.7 \%$ & $18 \%$ \\
\hline $\begin{array}{c}\text { Enterococcus } \\
\text { spp. }\end{array}$ & $16.9 \%$ & $5.1 \%$ & $4.1 \%$ & $18 \%$ \\
\hline S. aureus & $12.7 \%$ & - & $2.8 \%$ & $11 \%$ \\
\hline K. pneumoniae & $16.2 \%$ & $11.2 \%$ & $76 \%$ & $8 \%$ \\
\hline \multicolumn{5}{|c|}{ Microbiological Analysis } \\
\hline
\end{tabular}

\begin{tabular}{|c|c|}
\hline Studies & Specific Amoebic IgG Antibodies \\
\hline Present Study & $86 \%$ \\
\hline Jha et al(6) & $88 \%$ \\
\hline \multicolumn{2}{|c|}{ Amoebic Serology } \\
\hline
\end{tabular}

\begin{tabular}{|c|c|c|c|}
\hline Studies & $\begin{array}{c}\text { Anti-HIV } \\
\text { Antibodies }\end{array}$ & $\begin{array}{c}\text { HBsAG } \\
\text { Antibodies }\end{array}$ & $\begin{array}{c}\text { Anti-HCV } \\
\text { Antibodies }\end{array}$ \\
\hline Present Study & $2 \%$ & $6 \%$ & $4 \%$ \\
\hline Park et al(14) & $32 \%$ & - & - \\
\hline \multicolumn{4}{|c|}{ Viral Markers } \\
\hline
\end{tabular}

\section{Radiological Analysis}

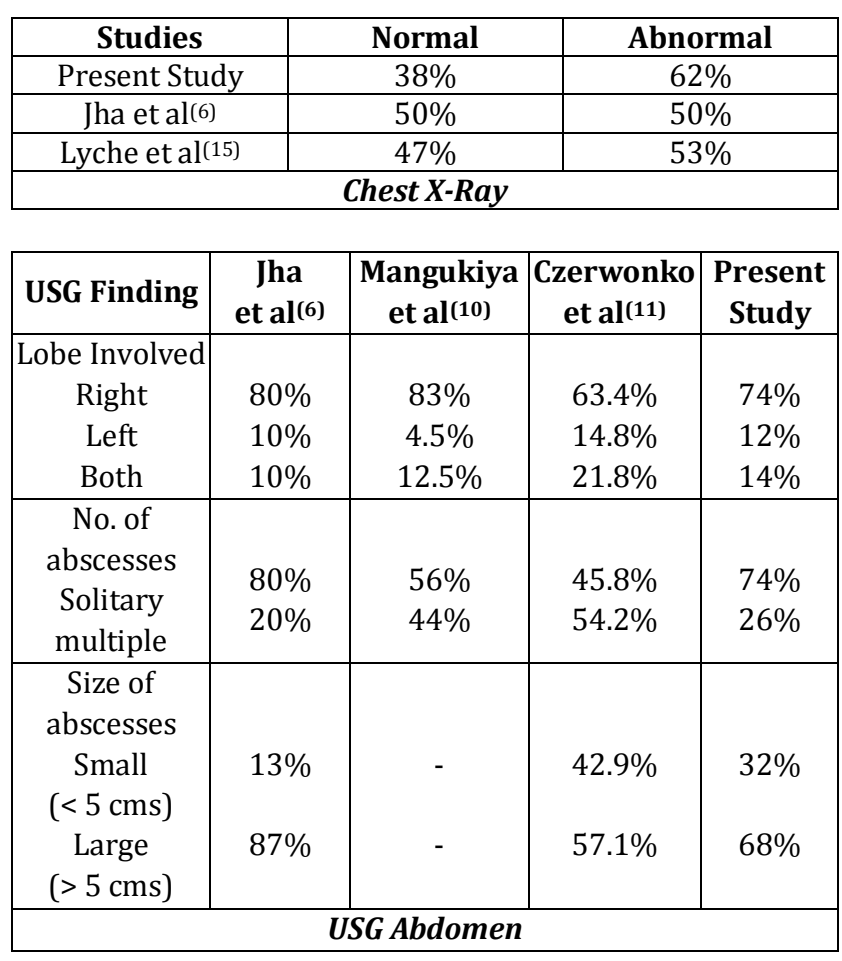

\begin{tabular}{|c|c|c|c|}
\hline Studies & $\begin{array}{c}\text { Ruptured } \\
\text { Liver Abscess }\end{array}$ & Septicaemia & $\begin{array}{c}\text { Pleural } \\
\text { Effusion }\end{array}$ \\
\hline Present Study & $18 \%$ & $10 \%$ & $2 \%$ \\
\hline Mangukiya et al(10) & $5 \%$ & - & - \\
\hline Chaudhary V et al(5) & $2 \%$ & - & - \\
\hline Peris et al (12) & - & $14.3 \%$ & $14.3 \%$ \\
\hline \multicolumn{4}{|c|}{ Complications } \\
\hline
\end{tabular}




\begin{tabular}{|c|c|c|c|}
\hline Studies & $\begin{array}{c}\text { Medical } \\
\text { (Antibiotics) }\end{array}$ & $\begin{array}{c}\text { USG-Guided } \\
\text { Pigtail } \\
\text { Drainage + } \\
\text { Antibiotics }\end{array}$ & $\begin{array}{c}\text { Open } \\
\text { Surgical } \\
\text { Drainage }\end{array}$ \\
\hline Present Study & $24 \%$ & $58 \%$ & $18 \%$ \\
\hline Peris et al(11) & $31.6 \%$ & $61.2 \%$ & $7.1 \%$ \\
\hline \multicolumn{3}{|c|}{ Treatment } \\
\hline
\end{tabular}

\begin{tabular}{|c|c|}
\hline Studies & Mortality Rate \\
\hline Mangukiya et al(10) & $1.5 \%$ \\
\hline Peris et al(12) & $8.2 \%$ \\
\hline Santos Rosa et al(3) & $20 \%$ \\
\hline Present study & $8 \%$ \\
\hline \multicolumn{2}{|c|}{ Outcome of Liver Abscess } \\
\hline
\end{tabular}

\begin{tabular}{|c|c|}
\hline Studies & Recurrence \\
\hline Present Study & $8 \%$ \\
\hline Chaudhary et al(5) & $8 \%$ \\
\hline \multicolumn{2}{|c|}{ Follow-Up and Recurrence } \\
\hline
\end{tabular}

\section{DISCUSSION}

- From our study it was concluded that about 3/4th of cases belonged to 4th to 6th decade. Males were more commonly affected than females. Alcoholism was the most consistent risk factor followed by diabetes mellitus. Most common presenting symptom and sign was abdominal pain and abdominal tenderness in right hypochondriac region respectively. Most common aetiology of liver abscess was amoebic followed by mixed amoebic and pyogenic infections. The most common organisms obtained from pus C/S from liver abscess were Enterococcus spp. and Escherichia coli. Abnormal chest $\mathrm{x}$-ray findings were recorded in $>50 \%$ of cases. USG showed majority of cases had large, solitary liver abscess in right lobe of liver. Most common complication observed was intraabdominal rupture of liver abscess for which open surgical drainage was performed. By following standard statistical protocols, all data will be collected and analysed to evaluate the clinicopathological aspects and response to standard institutional management strategies.

- Continuous data was summarised as Mean \pm SD (standard deviation of the mean), while categorical data was represented in numbers and percentages. Analyses were performed on SPSS software (windows version 17.0).

\section{CONCLUSION}

- $\quad$ Males are more commonly affected than females. About $3 / 4$ th of cases were found in 4 th to 6 th decade of life.

- Alcoholism was the most consistent risk factor followed by diabetes mellitus.

- Majority of patients presented within 7 days of onset of illness.

- Most common presenting symptom and sign was abdominal pain and abdominal tenderness in right hypochondriac region respectively.

- Most common aetiology of liver abscess was amoebic followed by mixed amoebic and pyogenic infections.
- The most common organisms obtained from pus $\mathrm{C} / \mathrm{S}$ from liver abscess were Enterococcus spp. and Escherichia coli.

- Abnormal chest $x$-ray findings were recorded in $>50 \%$ of cases.

- USG showed majority of cases had large, solitary liver abscess in right lobe of liver.

- Most common complication observed was intraabdominal rupture of liver abscess, for which open surgical drainage was performed.

- $\quad$ Recurrence was observed more following conservative management than other modalities.

\section{REFERENCES}

[1] Kapoor OP. Amoebic liver abscess. $1^{\text {st }}$ edn. Bombay: SS Publishers 1999.

[2] Perez JY Jr. Amoebic liver abscess: revisited. Philip J Gastroenterology 2006;2:11-3.

[3] Dos Santos-Rosa OM, Lunardelli HS, Ribeiro-Junior MAF, et al. Pyogenic liver abscess: diagnostic and therapeutic management. ABCD: Arquivos Brasileiros de Cirurgia Digestiva (São Paulo) 2016;29(3):194-7.

[4] Gopal LV, Rao VD, Srinu M, et al. A study on liver abscess in a tertiary hospital. IJGMP: Int J of Gen Med \& Phar 2014;3(1):1-4.

[5] Choudhary V, Chaudhary A. Clinico-pathological profile of liver abscess: a prospective study of 100 cases. International Surgery Journal 2016;3(1):26670.

[6] Jha AK, Das A, Chowdhury F, et al. Clinicopathological study and management of liver abscess in a tertiary care center. Journal of Natural Science, Biology and Medicine 2015;6(1):71-5.

[7] Mathur S, Gehlot RS, Mohta A, et al. Clinical profile of amoebic liver abscess. J Indian Acad Clin Med 2002;3(4):367-73.

[8] Chan KS, Chen CM, Cheng KC, et al. Pyogenic liver abscess: a retrospective analysis of 107 patients during a 3-year period. Japanese Journal of Infectious Diseases 2005;58(6):366-8.

[9] Kapadia S, Dattaroy D. Liver abscess. Indian Journal of Surgery 2002;6:511-9.

[10] Mangukiya DO, Darshan JR, Kanani VK, et al. A prospective series case study of pyogenic liver abscess: recent trands in etiology and management. Indian Journal of Surgery 2012;74(5):385-90.

[11] Czerwonko ME, Huespe P, Bertone S, et al. Pyogenic liver abscess: current status and predictive factors for recurrence and mortality of first episodes. HPB (Oxford): The Official Journal of the International Hepato Pancreato Biliary Association 2016;18(12):1023-30.

[12] Peris J, Bellot P, Roig P, et al. Clinical and epidemiological characteristics of pyogenic liver abscess in people 65 years or older versus people under 65: a retrospective study. BMC Geriatrics 2017;17(1):161.

[13] Kong H, Yu F, Zhang W, et al. Clinical and microbiological characteristics of pyogenic liver abscess in a tertiary hospital in East China. Medicine (Baltimore) 2017;96(37):e8050. 


\section{Jemds.com}

[14] Park WB, Choe PG, Jo JH, et al. Amebic liver abscess in HIV-infected patients, Republic of Korea. Emerging Infectious Diseases 2007;13(3):516-7.
Original Research Article

[15] Lyche KD, Jensen WA, Kirsch CM, et al. Pleuropulmonary manifestations of hepatic amebiasis. Western Journal of Medicine 1990;153(3):275-8. 